\title{
Microbial Community Structure in Soils Amended With Glyphosate-tolerant Soybean Residue
}

\author{
Mark Nye ${ }^{1}$, Nigel Hoilett ${ }^{2}$, Cliff Ramsier ${ }^{3}$, Peter Renz ${ }^{1}$, Richard P. Dick ${ }^{1, *}$ \\ ${ }^{1}$ School of Environment and Natural Resources, The Ohio State University, 2021 Coffey Rd, Columbus OH, 43210, USA \\ ${ }^{2}$ Department of Agricultural Sciences, Northwest Missouri State University, 800 University Dr, Valk 101, Maryville MO 64468, USA \\ ${ }^{3}$ Ag Spectrum, 428 East 11th Street, DeWitt IA, 52742, USA \\ *Corresponding author: dick.78@osu.edu
}

Received April 24, 2014; Revised June 11, 2014; Accepted June 12, 2014

\begin{abstract}
Glyphosate is a broad-spectrum herbicide used extensively worldwide to control broadleaf weeds in agriculture. Research suggests that repeated application causes a change in soil microbial properties which could be affecting soil quality and productivity. Although glyphosate is generally regarded as having relatively low environmental impact, after 10 or more years of widespread use, field observations by farmers and emerging research suggest that long-term glyphosate tolerant (GT) cropping is having cumulative and non-target effects on soils and crop productivity. There is very little information on the effects of GT residue when added to soils. Therefore, the objective was to determine shifts in the soil microbial community during GT residue decomposition in soils with and without a history of glyphosate exposure. Soybean residues from a simulated long-term GT cropping system were used in laboratory incubation. The experiment was a $2 \times 3 \times 4$ factorial design with 2 soils (with or without glyphosate), three residue types (leaf, stem,or root), and 4 soybean residue treatments (GT residue exposed to glyphosate with potassium salt carrier, GT residue exposed to glyphosate with isopropylamine salt carrier, untreated GT genotype, and untreated non-GT genotype). These soils were profiled using phospholipid fatty acid analysis to determine shifts in soil microbial community structure due to the addition of GT residue to soil. The results showed that microbial shifts during decomposition of GT soybean residue varied between soils with or without long-term exposure to glyphosate. There was also a trend that GT material that had been exposed to glyphosate cause a differential shift in the communities over GT residue that had not been exposed to glyphosate. Commercially available glyphosate formulations have two major types of salt carriers; potassium salt and isopropylamine salt which could be a factor besides glyphosate in affecting the chemistry of GT residues and subsequently microbial response during decomposition. However, the results showed that carrier did not significantly affect PLFA profiling in soils regardless of the soil's history of glyphosate exposure. Ratios of saturated to monounsaturated PLFAs are used as indicators of microbial stress. Our results showed that soil history of glyphosate exposure significantly affected microbial stress. There were also significant differences in stress between glyphosate residue treatments in soil with a history of glyphosate exposure.
\end{abstract}

Keywords: glyphosate, glyphosate-tolerant, PLFA, microbial ecology

Cite This Article: Mark Nye, Nigel Hoilett, Cliff Ramsier, Peter Renz, and Richard P. Dick, "Microbial Community Structure in Soils Amended With Glyphosate-tolerant Soybean Residue." Applied Ecology and Environmental Sciences, vol. 2, no. 3 (2014): 74-81. doi: 10.12691/aees-2-3-1.

\section{Introduction}

Glyphosate is a broad-spectrum, non-selective systemic herbicide that is very effective against broadleaf weeds and grasses. It was developed and patented by the Monsanto Corporation in the early 1970s. In 1996, genetically modified glyphosate-tolerant (GT) crops (Roundup Ready ${ }^{\circledR}$ ) were made commercially available, and in that year were grown on 1.7 million hectares worldwide. Since that time, the use of glyphosate to control weeds in agriculture has dramatically increased. The use of glyphosate and GT soybeans is widespread in Ohio and throughout the Midwest. In 2011, GT soybeans accounted for 94 percent of US soybean acreage, or 71.7 million acres [1]. Glyphosate has greatly reduced the use of other herbicides and is a critical component of reduced tillage systems [2], which improve surface water quality and soil water retention while reducing soil erosion and herbicide leaching in comparison to conventional tillage [3]. Glyphosate is generally regarded as having a low environmental impact and a low mammalian toxicity because it is water soluble and does not accumulate in food webs. Glyphosate is relatively non-volatile, adsorbs to clay particles as well as iron and aluminum oxides in soil, and thus has little potential to contaminate ground and surface water.

Research on the impact of GT cropping on soils has had mixed results. However, the majority of this research was done on short term glyphosate exposure. Since the commercial release of GT cropping systems in 1996, 
glyphosate has been in heavy use with several applications per year. It is important to investigate long-term effects of GT cropping, because it is likely that only after repeated glyphosate exposure would there be a shift in microbial communities. It has been shown that 2-4 applications of glyphosate can reduce $\mathrm{C}$ mineralization and increase glyphosate half-life when compared to a single application [4]. The degradation patterns of glyphosate have also been shown to change after repeated glyphosate applications [5]. This may suggest a shift in the microbial population toward microorganisms capable of metabolizing glyphosate. Reference [6] showed that repeated application of glyphosate can change the response of soil microorganisms over time. Field studies and unpublished data suggest that the effects on soil microbial communities can only be seen after long-term repeated application of glyphosate [7-13].

Glyphosate-tolerant plants may remain unaffected by the application of glyphosate-based herbicides. However, studies have shown that glyphosate application can have adverse effects on plants' nutrient uptake [14,15], and that that frequent applications of glyphosate may cause micronutrient deficiencies in GT and non-GT plants [8]. This has been attributed to the effect of glyphosate on the soil microbial community composition, which can change soil nutrient dynamics [12,13].

Most of the glyphosate applied is dispersed onto plant surfaces rather than directly onto the soil. However, the herbicide may come in contact with bare soil during spraying or by translocation through actively growing plants [16]. The rate at which soybeans can exude glyphosate from their roots can exceed 1000 ng per plant over the sixteen day period following application [17]. Because degradation of glyphosate in the soil is a result of microbial activity $[18,19]$, degradation rates of glyphosate can vary with the conditions that affect microbial activity, such as temperature and moisture content.

Glyphosate undergoes little to no metabolism in plants, and thus is eventually secreted into the soil. Root exudates from treated glyphosate-tolerant plants contain not only unbound glyphosate, but also elevated levels of carbohydrates and amino acids as compared to untreated plants [17]. As the herbicide residue in root exudates is degraded in the rhizosphere, the $\mathrm{C}$ present in glyphosate molecules is utilized by select fungi during their metabolic processes [20]. By this metabolic mechanism, glyphosatederived $\mathrm{C}$ is incorporated into cytoplasmic carbohydrates by certain species of fungi. The subsequent stimulation in growth of these fungi are possibly attributable not only to a novel source of $\mathrm{C}$ and nitrogen in the form of glyphosate, but also to the increase in carbohydrates and amino acids, as mentioned above.

A limited amount of research has been conducted on the decomposition of GT plant residues. Glyphosate use can significantly reduce crop residue decomposition. However, results were inconsistent and varied widely with weather and geography [21]. Furthermore, this effect was shown to be dependent on the location of residue, where decomposition rates were reduced for surface residues but not incorporated residues. It was also shown that decomposition rates were not significantly different between GT and non-GT crop varieties.

To our knowledge, there have been no studies on the effects of GT residue decomposition on soil microbial communities. Furthermore, few studies have investigated the effect of long-term GT cropping systems on microbial communities. Therefore, the objective of this study was to profile soil microbial communities using PLFA analysis during decomposition of GT residues added to soils with and without history of GT cropping.

\section{Materials and Methods}

\subsection{Soils}

Two soils were chosen, with one having had a history of GT cropping of $>10$ years $\left(\mathrm{GLY}^{+}\right)$, and the other having had no known history of exposure to glyphosate (GLY') (Table 1). The GLY' soil was a Blount silt loam (fine, illitic, mesic Aeric Epiaqualf). This soil was from an organically managed farm located in Delaware County, Ohio utilizing a continuous rotation; the previous five years were alfalfa-orchard grass-corn, oats-alfalfa-orchard grass, spelt-timothy-clover, and timothy-clover. The $\mathrm{GLY}^{+}$soil was a Bennington silt loam (fine, illitic, mesic Aeric Epiaqualf) from a farm in Knox County, Ohio practicing a no-till corn-soybean rotation (soybeans were GT). Glyphosate was applied up to three times per year while growing soybeans, and once per year while cultivating corn. Bennington and Blount silt loams are taxonomically identical except that the Blount has greater calcium carbonate concentration in the $\mathrm{C}$ horizon, a depth not sampled in this experiment.

Soil samples were collected with probes $(2.5 \mathrm{~cm} \mathrm{x} 20$ cm) randomly at each of the field sites in January 2012. The samples were inspected in the lab, where stones, large pieces of organic matter and roots were removed. The soils from each site were homogenized, sieved to pass 2 $\mathrm{mm}$ mesh size, and stored in sealed plastic bags at $4^{\circ} \mathrm{C}$.

Table 1. Soil pH, C content, and texture

Table 1. Soil pH, C content, and texture
\begin{tabular}{|c|c|c|c|c|c|c|}
\hline \multirow{2}{*}{ Symbol } & \multirow{2}{*}{ Soil Type } & \multirow{2}{*}{$\mathrm{pH}$} & \multirow{2}{*}{ Total C } & \multicolumn{3}{|c|}{ Soil Texture } \\
\cline { 5 - 8 } & & & Clay & Silt & Sand \\
\hline GLY-------------\%--------- \\
\hline GLY+ & Blount Silt Loam & 6.95 & 1.47 & 41 & 48 & 11 \\
\hline
\end{tabular}

\subsection{Plant Residue}

The soybean residues used in this experiment came from a greenhouse study where corn (Zea mays) and soybeans (Glycine max) were grown in rotation for 8 growth periods that ran 58 days each. The two glyphosate treatments used had different carrier molecules: Powermax ${ }^{\circledR}$ (Monsanto, Inc., St. Louis, MO) potassium salt of $\mathrm{N}$-(phosphonomethyl)glycine and Cornerstone ${ }^{\circledR}$ (AgriSolutions, Brighton, IL) isopropylamine salt of $\mathrm{N}$ (phosphonomethyl) glycine. Additionally, there were GT and non-GT plant controls to which no glyphosate was applied. At the end of each period, plant biomass was harvested, dried, and stored in paper bags at ambient temperature.

Soybean residues from each box were separated into leaf, stem, and roots. All tissues were dried and weighed. Roots were rinsed with distilled water to remove any excess soil. The distribution of leaf, stem and root residues calculated from the dry weights of the harvested plants in the greenhouse study was $32.7: 51.8$ : 15.5 leaf, stem, and root, respectively. This was similar to the distribution 
reported in [22]. This distribution of leaf, stem, or root residue was used in the incubation study described below.

\subsection{Incubation}

The incubation study used a 2 (soils) x 3 (residue types) x 4 (glyphosate treatments) factorial design: two soils (with and without a history of long-term GT cropping), three soybean residue types (leaf, stem, root), and four glyphosate residue treatments the greenhouse experiment:

1. Powermax ${ }^{\circledR}$-treated GT soybean plants

2.Cornerstone ${ }^{\circledR}$-treated GT soybean plants

3. untreated GT soybean plants

4. untreated non-GT plants

Prior to incubation, $28 \mathrm{~g}$ of soil was weighed in each glass sample jar (small Whatman jar, 15mm radius). Water was added by weight to attain $66 \%$ field capacity, and the total weight of each sample jar containing soil was recorded for future soil moisture adjustments. Moisture content of the samples was maintained gravimetrically at $66 \%$ field capacity by adding distilled water as needed after each destructive sampling day.

Into each $28 \mathrm{~g}$ soil sample was added either $132 \mathrm{mg}$ leaf residue, $83 \mathrm{mg}$ stem residue, or $39 \mathrm{mg}$ root residue. The jars were closed with a plastic cap and shaken briefly to ensure thorough distribution of residue throughout each soil sample. Jars were placed in plastic storage bins, each with an open beaker of distilled water, and were then covered with plastic wrap. The plastic wrap was punctured several times with a hypodermic needle, and the bins were then placed in an incubator at $22^{\circ} \mathrm{C}$. Microcosms were destructively sampled in triplicate at 3, 7, and 30 days. After sampling, each jar was sealed with a plastic cap and stored at $-20^{\circ} \mathrm{C}$ until phospholipid fatty acid analysis.

\subsection{PLFA Analysis}

A modified version of the phospholipid fatty acid (PLFA) extraction method as described by [23] was used in this experiment. Total lipids were extracted from $2 \mathrm{~g}$ of soil from each sample using a chloroform: methanol: aqueous citrate buffer (1:2:0.8) extractant [24]. The total lipid extract was then separated into neutral lipids, glycolipids, and phospholipids using silicic acid columns. Phospholipids were then subjected to alkaline methanolysis and dried under $\mathrm{N}_{2}$ in a $35^{\circ} \mathrm{C}$ heating block. The dry sample was reconstituted in $170 \mu \mathrm{l}$ of $1: 1(\mathrm{v} / \mathrm{v})$ hexane: methyl tert-butyl ether (MTBE), transferred to GC vials, and combined with an internal standard (30 $\mu \mathrm{l}, 0.01$ M C19:0-ME in 1:1 Hexane: MTBE).

PLFA detection and quantification were performed on Agilent N6890 gas chromatograph (Agilent Technologies, USA) equipped with an Agilent 7683 Series Injector and a flame ionization detector (FID). The MIDI System, PLFAD1 protocol (MIDI Inc., Newark, DE, USA), in combination with the Agilent ChemStation Software was then used to measure peak area (response) and to identify PLFAs. These PLFAs were associated with a specific lipid biomarker.

\subsection{Statistical Analysis}

PLFA concentrations were analyzed using both absolute concentration (nmol g-1 soil) and relative concentration (percent of total moles of PLFA). Seventeen
PLFAs were used for analysis. PLFAs used as biomarkers for functional groups were summed, and statistical analysis was performed on totals PLFA amounts representing six functional taxonomic groups: gram positive bacteria, gram negative bacteria, fungi, actinomycetes, arbuscular mycorrhizal fungi (AMF), and eukaryotes. The ratio of saturated to monounsaturated fatty acids (SAT/MONO) was calculated as an indicator of microbial stress $[25,26]$.

Two-way analysis of variance (ANOVA) and StudentNewman-Keuls stepwise multiple comparison test were used to evaluate the significance of soil glyphosate history, glyphosate treatment, residue type, and sampling day for each functional taxonomic group. This analysis was also used to evaluate differences in microbial stress between soils and glyphosate treatments. ANOVA analyses were conducted using SAS 9.0 software.

Non-metric multidimensional scaling (NMS) was performed using PC-ORD based on Sørensen distance. Two hundred and fifty runs were conducted with real data and compared to 250 randomized runs. A stability criterion of 0.00001 was used. NMS was performed on both the absolute and relative concentration data sets (nmol g-1 soil and percent of total moles of PLFA). Prior to NMS, the data were transformed using a monotonic square root transformation to improve normality and reduce the coefficient of variation among PLFAs.

\section{Results}

\subsection{Soil Type}

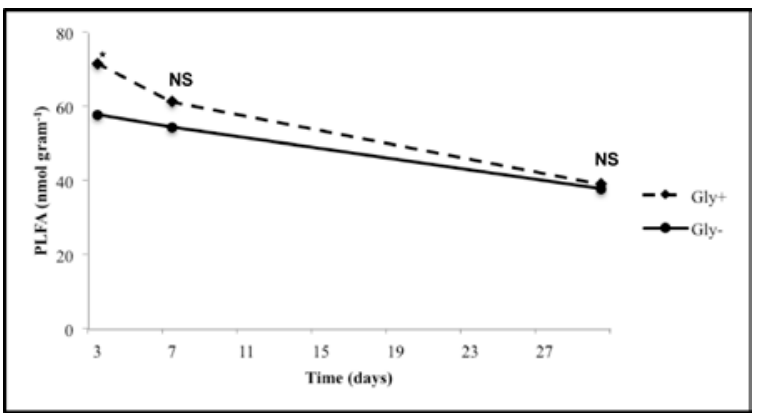

Figure 1. Time course PLFA concentrations for gram positive bacteria averaged over soil type.

The most significant effects on PLFA diversity were due to the soil source, where the main difference was glyphosate-tolerant (GT) and non-GT cropping. All six functional taxonomic groups, were significantly different at various sampling dates over the course of the experiment due to soil type. PLFA concentrations for gram positive bacteria showed a significantly higher concentration in GLY+ soil when compared to GLY-soil at Day 3 than at Day 30 (Figure 1). Both concentrations decreased over the course of the incubaiton, however, the rate of decrease was faster for gram positive bacteria in the GLY+ soil than in the GLY- soil. PLFA concentrations for gram negative bacteria were significantly different between the two soils at all three sampling days and decreased throughout the course of the incubation (Figure 2). Concentrations of gram negative PLFA in the two soils decreased at approximately the same rate. PLFA concentrations for fungi were not 
significantly different at Day 3 or Day 7, but were significantly different at Day 30 (Figure 3). The rate of decrease was faster for the fungal PLFAs in GLY- soil than in GLY+ soil.

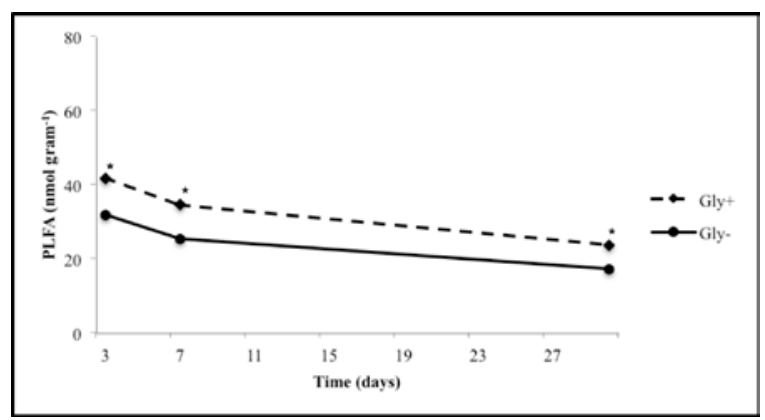

Figure 2. Time course PLFA concentrations for gram negative bacteria averaged over soil type.

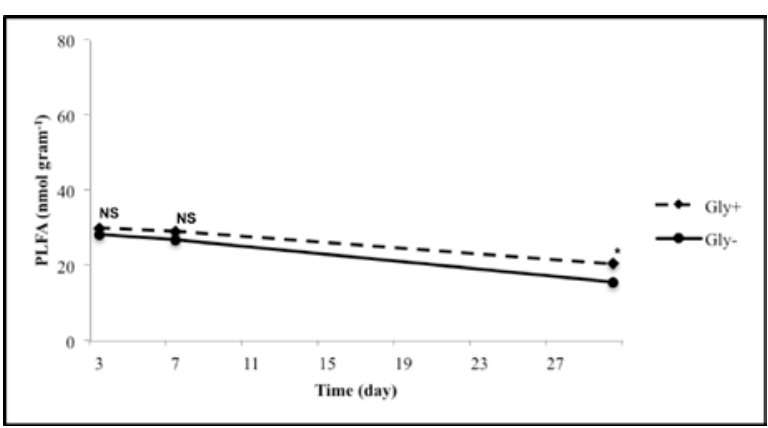

Figure 3. Time course PLFA concentrations for fungi averaged across soil type.

The only significant difference due to residue glyphosate treatments (averaged across all residue types) in the GLY+ soil were found on Day 7 in the eukaryote group (Figure 4). In this case, there was a significant difference in PLFA concentrations between soils amended with Powermax treated residue and soils amended with untreated residues (GT and non-GT). However, there was no significant difference between the Powermax and Cornerstone treatments, nor was there a significant difference between the Cornerstone treatments and the untreated samples. There were no significant differences between the individual residues (averaged across all treatments) in the GLY+ soil in any functional taxonomic group on any sampling day (data not shown).

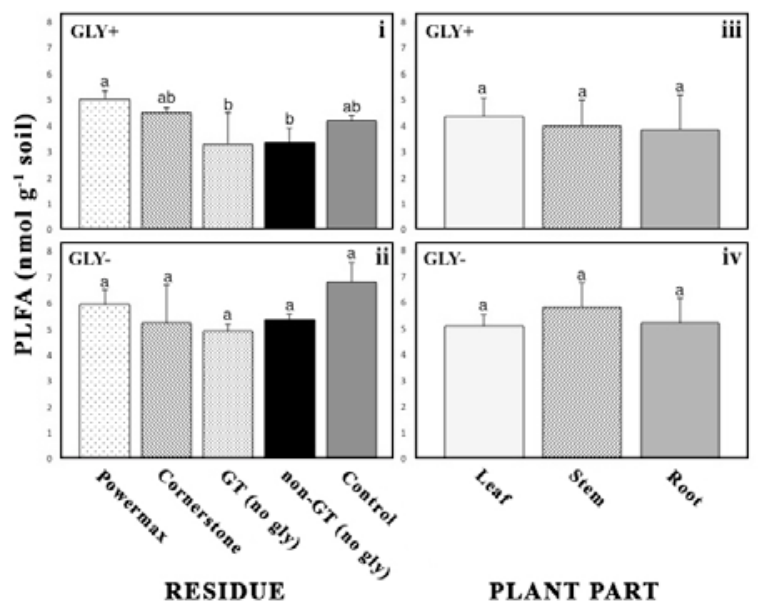

Figure 4. PLFA biomarker concentrations for eukaryotes, Day 7. Bars within soil having the same letter are not significantly different at $\mathrm{P}<0.05$.
Figures i and ii are averaged over plant parts. Figures iii and iv are averaged over residue glyphosate treatments.

In contrast GLY+ soil, there were more treatment effects on the GLY- soil. For this soil, significant differences were found on Day 3 for the fungal PLFA values averaged across residues and across treatments (Figure 5). There was a significant difference between values averaged across all treatments of the fungal PLFA concentration for leaf residue samples when compared to the stem and root residue samples. There was also a significant difference in values averaged across all residues of the fungal PLFA concentration for the samples amended with Powermax treated residues when compared to the other treatments. Within residue types, there were significant differences in fungal PLFA concentrations 1) between Powermax and the other treatments for leaf residue samples and 2) between the glyphosate treated and non-treated root residue samples. These significant differences were not found in the Day 7 and Day 30 samples. Similarly, significant differences were found on Day 3 for the actinomycete PLFA biomarkers (Figure 6), and like the fungal PLFAs, the significant differences were not found on Days 7 or 30.

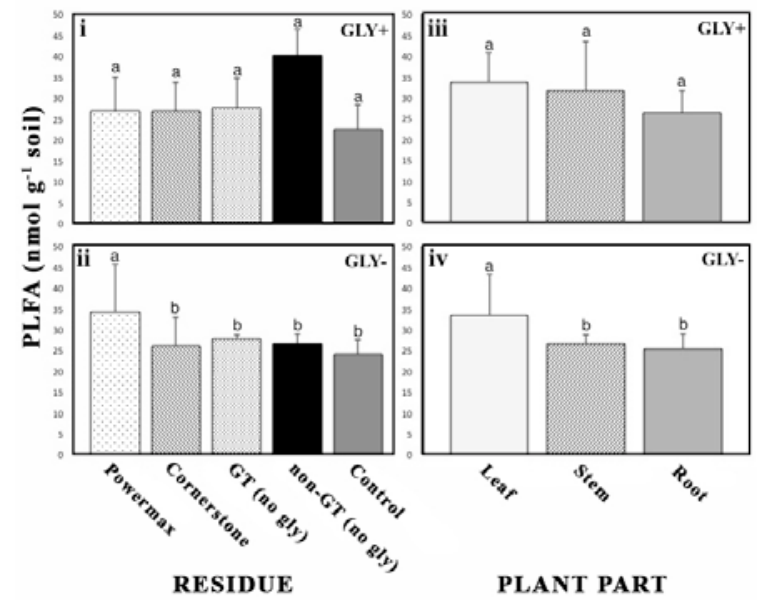

Figure 5. PLFA biomarker concentrations for fungi, Day 3. Bars within soil having the same letter are not significantly different at $\mathrm{P}<0.05$. Figures i and ii are averaged over plant parts. Figures iii and iv are averaged over residue glyphosate treatments.

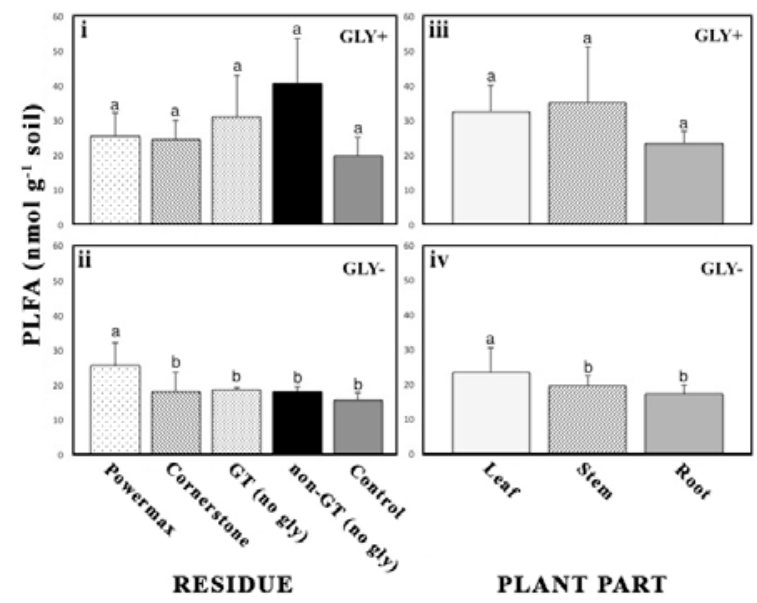

Figure 6. PLFA biomarker concentrations for actinomycetes, Day 3. Bars within soil having the same letter are not significantly different at $\mathrm{P}<0.05$. Figures $\mathrm{i}$ and ii are averaged over plant parts. Figures iii and iv are averaged over residue glyphosate treatments. 


\subsection{Ordination Analysis}

Clustering of community PLFA profiles (all PLFAs) was distinct at Day 3 and Day 30 (Figure 7), but the soils were closer by Day 30 than at Day 3. Within soil types, the clustering patterns varied due to sampling day and due to glyphosate residue treatment. For example, root residue samples $n$ the GLY- soil showed clustering of profiles by sampling day (Figure 8). Days 3 and 7 are clustered more closely together than Day 30. In contrast, this effect is not found in samples taken from soil with stem residue in GLY+ soil. However, in the GLY-soil, the controls (no residue) are clearly separated; whereas in the GLY+ soil, the controls are clustered closely together. Furthermore, on Day 30 (the end of the incubation), the GLY- soil showed clustering of profiles from soils amended with untreated plant residues (GT and non-GT), an effect not found in the GLY+ (Figure 9). Clustering was not distinct in either soil due to residue type (leaf, stem, root) (data not shown).
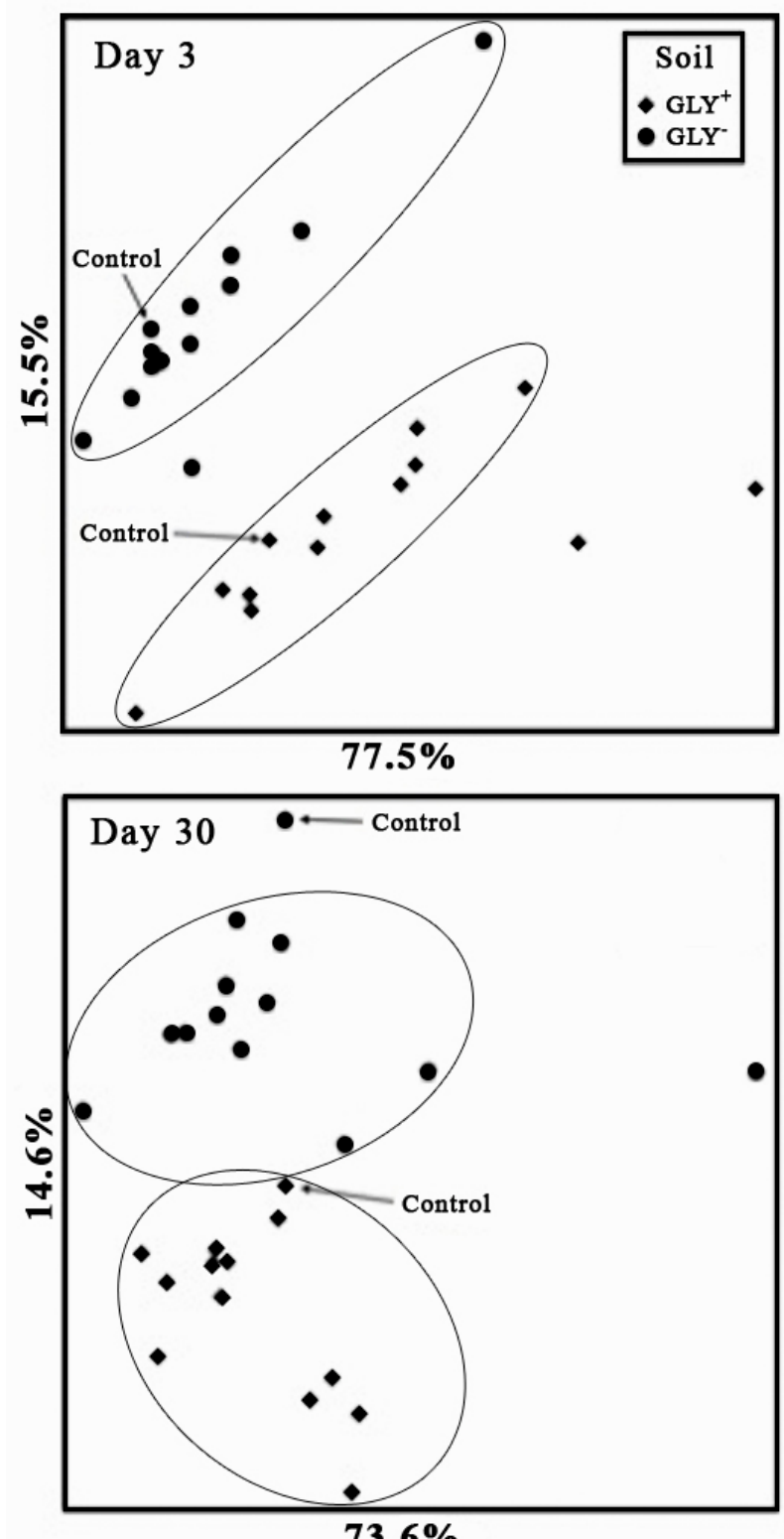

Figure 7. Ordination plots of all PLFA profiles from all treatments for both soils on Day 3 and Day 30. Each point is the mean (three replications) of PLFA community profile for a given soil-residuetreatment combination.
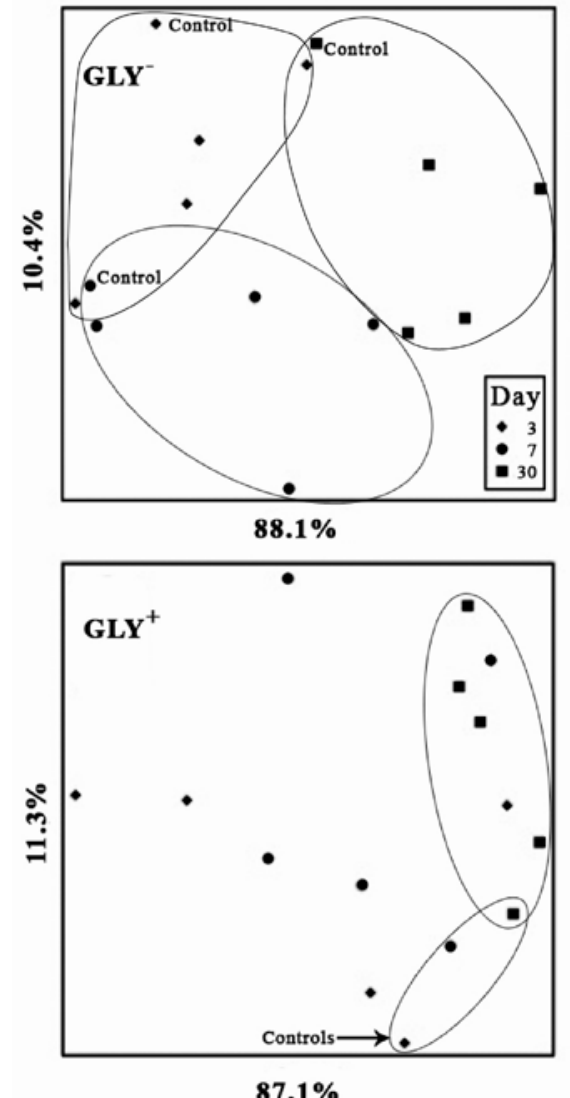

Figure 8. Ordination plot of PLFA profiles from root in soil with and without known history of glyphosate exposure amended with root or stem residue.
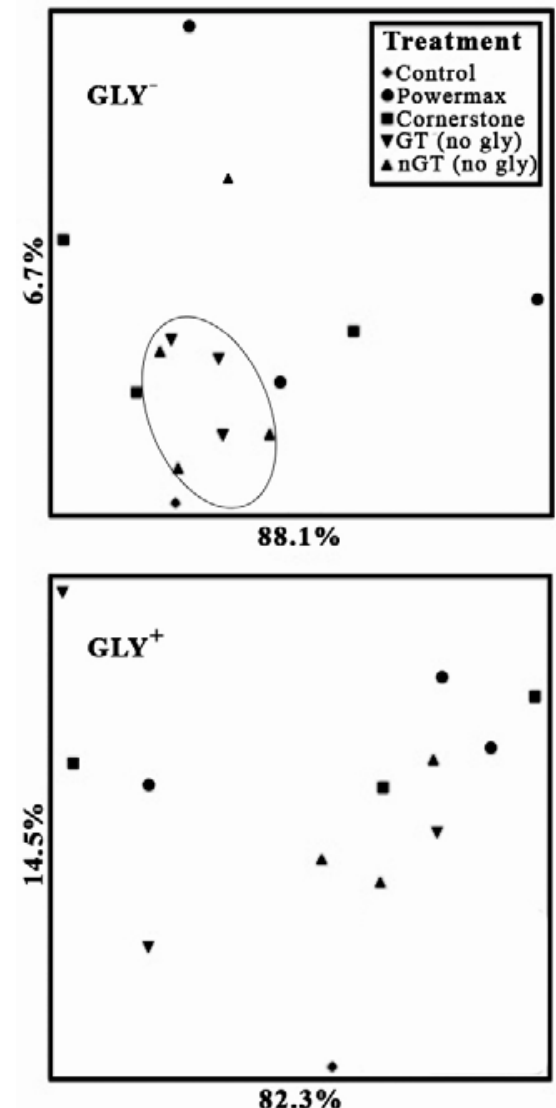

Figure 9. Ordination plot of PLFA profiles from Day 30 of all residue treatments in soil with and without known history of glyphosate exposure. 


\subsection{Microbial stress}

Significant differences were found between the two soil types for microbial stress indicators when averaged across all treatments. The GLY+ soil had greater stress than the GLY - soil at Day 3 and Day 30 (Figure 10). Significant differences were found in the SAT/MONO ratio due to residue glyphosate treatments in the GLY+ soil, but not in the GLY- soil (Figure 11). Additionally, there was a significant difference in the GLY+ soil between the control (no residue) and all other treatments that was not found in the GLY- soil. There were significant differences in stress indicators due to sampling day in both soils (data not shown). The SAT/MONO ratio was significantly higher at Day 3, then decreased by Day 7 and then increased again by Day 30 .

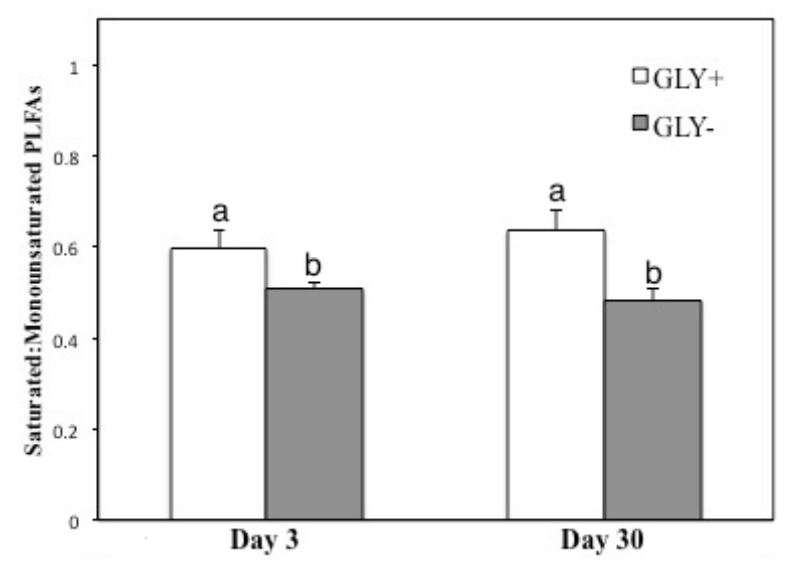

Figure 10. PLFA ratios indicating microbial stress averaged over all treatments at Day 3 and Day 30. Bars within soil having the same lowercase letter are not significantly different at $\mathrm{P}<0.05$.

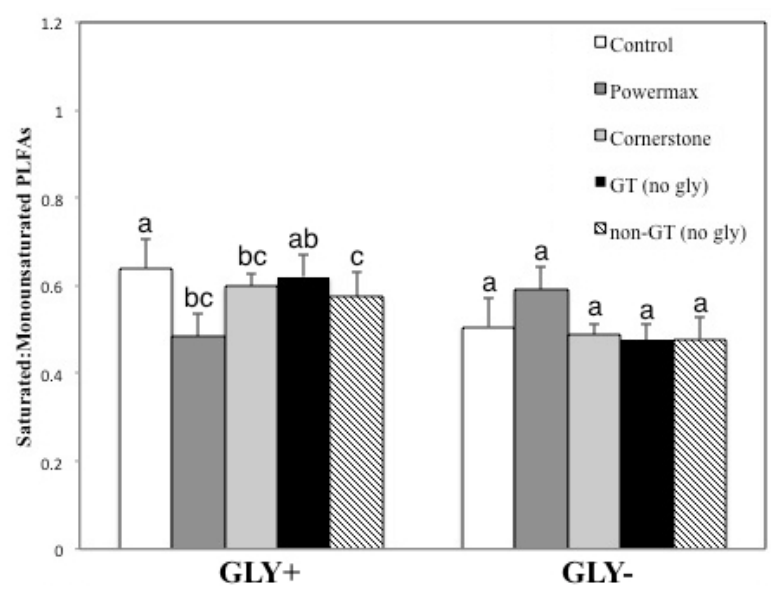

Figure 11. PLFA ratios indicating microbial stress averaged over the course of 30 day incubation. Bars within soil having the same lowercase letter are not significantly different at $\mathrm{P}<0.05$

\section{Discussion}

The most apparent effect on PLFA profiles was soil history of glyphosate exposure. This suggests that longterm repeated glyphosate application has an affected soil microbial communities. This is supported by ordination plots of community PLFA profiles where PLFA profiles clustered by soil type. This grouping is more evident early in the incubation (Day 3), and while the groups move closer to one another later in the incubation (Day 30), the grouping and separation of groups is still distinct between the GLY+ and GLY- soils (Figure 7). Furthermore, it is clear on Day 30 that the profiles of amended GLY-soils move further from the non-amended control. This suggests that the initial response of the microbial community during plant residue decomposition differs initially between the two soils, but that this effect diminishes over time. The effect was shown regardless of residue type or the glyphosate treatment applied to the growing plants.

Our findings are in contrast with studies that have shown that GT cropping under field conditions has little to no effect on soil microorganisms, [27,28,29,30]. However, none of these studies were done on soils that had been under long-term GT management, and all were short-term studies. Additionally, our residues were derived from an experiment conducted under greenhouse conditions rather than field conditions. Other studies of soils that received glyphosate under long-term GT management have not shown shifts in fatty acid profiles [5,31,32]. However, these studies had direct application of glyphosate to soil over short time periods rather than decomposition of glyphosate-exposed plant residues in soils with and without long-term GT cropping. Our study showed an initial difference between the soils with and without exposure to glyphosate and the differential response of the two soils to the amendment during the decomposition. The shift and distinct response were most prevalent in the GLY-soil. This would suggest that the GLY+ soil already had a community primed for glyphosate residues, whereas the GLY-soil was starting to change in response to those treatments.

Ordination analysis showed that, within each soil type, there are differences in the microbial community PLFA profiles for experimental factors (time, glyphosate treatment of residue, residue type). The GLY- soil showed distinct clustering of communities by sampling day within the incubation, regardless of the glyphosate treatment applied to the plant residue. This was consistent with [31], that studied forest soils and found clustering of PLFA profiles regardless of glyphosate application under field conditions. PLFA profiles of samples from Days 3 and 7 clustered more closely to one another than Day 30 (Figure 8). This was most evident in communities in soil samples to which root residue was applied. This effect was not found in the GLY+ soil for any residue. This suggests that the microbial communities in the soil with a history of long-term glyphosate exposure have adapted to GT residues and thus show fewer microbial responses during decomposition than the GLY- soil. This was shown in GLY- soil, where there was no clustering of Day 3 or Day 7 communities, but there was clustering at Day 30. This was the opposite for GLY+ soil which presumably was primed for these residues. Furthermore, despite the clear separation of profiles over sampling day in the GLY- soil, the controls (no residue) were very clearly separated from one another. This is in contrast to the GLY+ soil, where only Day 30 clustered, but the controls from all three days were clustered closely together (Figure 8).

The effect of glyphosate treatment previously applied to plants was only found at the end of the incubation (Day 30) in the GLY- soil (Figure 9). The PLFA profiles in samples to which untreated plant residue (GT and non-GT) was applied and the control (no residue) were more closely clustered and separated from the glyphosate treated 
residues. This effect was found across the means of all residues, regardless of whether the residue was GT or nonGT. No such grouping was see in the GLY+ soil. This result is consistent with [21], who found no significant difference between the decomposition of GT and non-GT corn and soybeans. No such grouping was found in the GLY+ soil. This suggests that the microbial communities in the GLY+ soil respond indiscriminately to the addition of treated/untreated plant residues, whereas the communities in the GLY- soil have more distinct microbial profiles between glyphosate treated and untreated residues. Neither soil shows any clear separation between GT and non-GT untreated residues. This would be consistent with [33], that there are no substantive differences in the nutritional value between GT and nonGT residues. A strong trend in our data suggests that it is the exposure to glyphosate and not the GT plant material itself that causes microbial responses.

The dominant PLFA markers changed over the incubation, with differential shifts in the PLFAs profiles between the GLY+ and the GLY- soils. For each functional group analysed, the initial PLFA concentration was higher in the GLY+ soil than in the GLY- soil. The PLFA concentrations representing each functional taxonomic group decreased over the course of the incubation. However, the changes over time varied between the two soils. Statistically significant differences were present between the PLFA concentrations in the two soils at different time points for different functional taxonomic groups. This suggested that soil history of glyphosate exposure was important in controlling microbial response to GT residues added to soils.

The concentration of gram positive bacterial PLFAs were significantly different between the two soils on Day 3 , and although the concentration in both soils decreased by Days 7 and 30, there were no significant differences at these days (Figure 1). This was the same for fungal PLFAs except that significant differences were only seen at Day 30 (Figure 3). In contrast, gram negative bacterial PLFAs were significantly different between the two soils at all time points (Figure 2). In all three functional groups, the concentration of PLFAs were higher in the GLY+ soil than in the GLY - soil.

Our results are consistent with [31]. PLFA markers for bacteria and fungi decreased over the 30 day incubation, and the differences in PLFA community profiles are more strongly tied to sampling date rather than herbicide treatment. However, [31] did not address the potential effects of long-term GT cropping on PLFA profiles. There may be differences because they studied forest soils rather than agricultural soils.

Significant differences between glyphosate treatments (Powermax, Cornerstone, untreated GT, untreated non-GT residues) were not found in the GLY+ soil, with the following exception: eukaryote PLFA markers were significantly different (averaged across all residues) between Powermax treated residues and either of the untreated residues on Day 7 (Figure 4). However, for this group, there was no significant difference between the samples with Cornerstone treated residues and the samples with untreated residues, nor between the Cornerstone and Powermax treated residues. Nonetheless, based on PLFA biomarkers across all functional groups, the data suggests limited microbial response in the GLY+ soil due to GT residues during decomposition. In the GLY- soil, there were two cases of significant differences between functional group PLFA concentrations due the residue glyphosate treatments, both of which were found on Day 3. In this case, the groups were fungi (Figure 5) and actinomycetes (Figure 6). PLFA markers for each group were significantly different in samples with Powermax treated residues compared to all other treatments.

Neither soil showed a significant difference in PLFA concentrations for any functional group between samples with Powermax treated residue and samples with Cornerstone treated residue (data not shown). This suggests that, regardless of soil history of glyphosate exposure, there is no difference in microbial response due to carrier molecules, which were a K salt with Powermax and an isopropylamine salt with Cornerstone. We found no other research on the effects of glyphosate carriers on microorganisms with which to compare our own research.

As was the case with the differences between glyphosate treatments, there were only a limited number of significant differences between residues (root, stem, leaf) when averaged across all glyphosate residue treatments (treated and untreated, GT and non-GT) within a given soil. These significant differences were found in the GLY- soil on Day 3 only. For concentrations of fungal PLFAs, there was a significant difference on Day 3 between leaf residue amended soil compared to stem and root amended soils (which were not significantly different from each other) (Figure 5). An identical result was observed on Day 3 for concentrations of PLFAs associated with actinomycetes (Figure 6). These results suggest that although we found these limited instances of specific differences between residues, it is difficult to assess the relevance of these differences, because under field conditions, residues would not be segregated from one another.

The microbial stress indicators we used showed significant differences in microbial stress between the two soils at Day 3 and at Day 30. Differences in stress due to sampling day, glyphosate residue treatment, and residue type all varied between GLY+ and GLY- soils. Stress was greater across all residue treatments at all sampling days for the GLY+ soil than the GLY- soil.

There were several treatment comparisons that did not affect PLFA profiling or stress levels within either soil type. Plant residue type (leaf, stem, root) showed no differential response across the other treatments. This was also the case for GT vs. non-GT soybean residue that had not been exposed to glyphosate. This is likely because major constituents of plant residues such as lignin, cellulose and other compounds which are dominant in controlling decomposition and microbial responses are quite similar between these varietal genotypes. The other comparison of glyphosate carrier also had no significant differences in PLFA profiling or stress levels. Although this should be investigated more thoroughly, it seems that the glyphosate molecule in plant residue is more important in affecting microbial response. The latter point above was detected in the soil that had not been exposed to GT cropping. Overall, the GLY- soil had a number of responses that were temporally meaningful, whereas the GLY+ soil showed very little response to any of the soil treatments. 


\section{Acknowledgement}

This research was generously supported by AgSpectrum and The Ohio State University Environmental Science Graduate Program.

\section{References}

[1] Grube, A. USEPA Pesticide Industry Sales and Usage Report. 2011.

[2] Young, B.G., Changes in herbicide use patterns and production practices resulting from glyphosate-resistant crops. Weed Technol. 20: 301-307. 2006.

[3] Fawcett, R.S., B.R. Christensen, and D.P. Tierney. The impact of conservation tillage on pesticide runoff into surface water: A review and analysis J. Soil Water Conserv. 49: 126-135. 1994.

[4] Andréa, M.M. de. Influence of repeated applications of glyphosate on its persistence and soil bioactivity. Pesq. Agropec. Bras. 38: 1329-1335. 2003.

[5] Lancaster, S.H., E.B. Hollister, S.A. Senseman and T.J. Gentry. Effects of repeated glyphosate applications on soil microbial community composition and the mineralization of glyphosate. Pest Manag. Sci. 66: 59-64. 2010.

[6] Quinn J.P., J.M.M. Peden, and R.E. Dick. Glyphosate tolerance and utilization by the microflora of soils treated with the herbicide. Appl. Microbiol. Biotechnol. 29: 511-516. 1988.

[7] Fernandez, M.R., F. Selles, D. Gehl, R. Depauw and R. Zentner. Crop production factors associated with Fusarium head blight in spring wheat in eastern Saskatchewan. Crop Sci. 45: 1908-1916. 2005.

[8] Huber, D. Strategies to ameliorate glyphosate immobilization of manganese and its impact on disease. Phytopathology. 97: S168S168. 2007.

[9] Locke, M., R. Zablotowicz and K. Reddy. Integrating soil conservation practices and glyphosate-resistant crops: Impacts on soil. Pest Manag. Sci. 64: 457-469. 2008.

[10] Fernandez, M.R., R.P. Zentner, P. Basnyat, D. Gehl, F. Selles and D. Huber. Glyphosate associations with cereal diseases caused by Fusarium spp. in the canadian prairies. Eur. J. Agron. 31: 133-143. 2009.

[11] Johal, G. and D. Huber. Glyphosate effects on diseases of plants. Eur. J. Agron. 31: 144-152. 2009.

[12] Kremer, R. and N. Means. Glyphosate and glyphosate-resistant crop interactions with rhizosphere microorganisms. Eur. J. Agron. 31: 153-161. 2009.

[13] Yamada, T., R. Kremer, P. Castro and B. Wood. Glyphosate interactions with physiology, nutrition, and diseases of plants: Threat to agricultural sustainability? preface. Eur. J. Agron. 31: 111-113. 2009.

[14] Eker, S., L. Ozturk, A. Yazici, B. Erenoglu, V. Romheld and I. Cakmak. Foliar-applied glyphosate substantially reduced uptake and transport of iron and manganese in sunflower plants. J. Agric. Food Chem. 54: 10019-10025. 2006.

[15] Neumann, G., S. Kohls, E. Landsberg, K. Souza, T. Yamada and V. Roemheld. Relevance of glyphosate transfer to non-target plants via the rhizosphere. Journal of Plant Dis. Protect. 20: 963969. 2006.

[16] Coupland, D. and J. Caseley. Presence of ${ }^{14} \mathrm{C}$ activity in root exudates and guttation fluid from agropyron-repens treated with ${ }^{14}$ C-labeled glyphosate. New Phytol. 83: 17-22. 1979.
[17] Kremer, R., N. Means and S. Kim. Glyphosate affects soybean root exudation and rhizosphere micro-organisms Int. J. Environ. Anal. Chem. 85: 1165-1174. 2005.

[18] Rueppel, M., B. Brightwell, J. Schaefer and J. Marvel. Metabolism and degradation of glyphosate in soil and water. J. Agric. Food Chem. 25: 517-528. 1977.

[19] Shushkova, T.V., G.K. Vasilieva, I.T. Ermakova, and A.A. Leontievsky. Sorption and microbial degradation of glyphosate in soil suspensions. Appl. Biochem. Microbiol. 45: 599-603. 2009.

[20] Charnay, M. P., C. Mougin, A. Farrugia, and E. Barriuso. Incorporation of pesticides by soil micro-organisms as a way of bound residues formation. Environ. Chem. Lett. 2: 27-30. 2004.

[21] Powell, J.R., D.J. Levy-Booth, R.H. Gulden, W.L. Asbil, R.G. Campbell, K.E. Dunfield, A.S. Hamill, M. Hart, S. Lerat, R.E. Nurse, K. Pauls, P. Sikkema, C.J. Swanton, J.T. Trevors and J.N. Klironomos. Effects of genetically modified, herbicide-tolerant crops and their management on soil food web properties and crop litter decomposition. J. Appl. Ecol. 46: 388-396. 2009.

[22] Bergersen, F.J., G.L. Turner, R.R. Gault, M.B. Peoples, L.J. Morthorpe, J. Brockwell, Contributions of Nitrogen in Soybean Crop Residue to Subsequent Crops and to Soil. Aust. J. Agric. Res. 43: 155-169. 1992

[23] Frostegård, A. and E. Bååth. The use of phospholipid fatty acid analysis to estimate bacterial and fungal biomass in soil. Biol. Fert Soils. 22: 59-65. 1996.

[24] Bligh, E.G., and W.J. Dyer. A Rapid Method of Total Lipid Extraction and Purification. Can. J. Biochem. Physiol. 37: 911917. 1959,

[25] Larkin, R.P. Characterization of soil microbial communities under different potato cropping systems by microbial population dynamics, substrate utilization, and fatty acid profiles. Soil Biol. Biochem. 35: 1451-1466. 2003

[26] Moore-Kucera, J and R.P. Dick. PLFA profiling of microbial community structure and seasonal shifts in soils of a Douglas-fir chronosequence. Microb. Ecol. 55: 500-511. 2008.

[27] Lupwayi, N.Z., and R.E. Blackshaw. Soil microbiology in glyphosate-resistant corn cropping systems. Agron. J. 104: 10411048. 2012.

[28] Hart, M.R. and P.C. Brookes. Soil microbial biomass and mineralization of soil organic matter after 19 years of cumulative field applications of pesticides. Soil Biol. Biochem. 28: 1641-1649. 1996.

[29] Wardle, D.A., and D. Parkinson. Influence of the herbicide glyphosate on soil microbial community structure. Plant and Soil. 122: 29-37. 1990.

[30] Wardle, D.A., and D. Parkinson. The influence of the herbicide glyphosate on interspecific interactions between four soil fungal species. Mycol. Res. 96: 180-186. 1992.

[31] Ratcliff, A.W., M.D. Busse, C.J. Shestak. Changes in microbial community structure following herbicide (glyphosate) additions to forest soils. Appl. Soil Ecol. 34: 114-124. 2006.

[32] Weaver, M.A., L.J. Krutz, R.M. Zablotowicz, and K.N. Reddy. Effects of glyphosate on soil microbial communities and its mineralization in Mississippi soil. Pest. Manag. Sci. 63: 388-393. 2007.

[33] Duke, S.O., J. Lydon, W.C. Koskinen, T.B. Moorman, R.L. Chaney, and R. Hammerschmidt. Glyphosate effects on plant mineral nutrition, crop rhizosphere microbiota, and plant disease in glyphosate-resistant crops. J. Ag. Food Chem. 60: 10375-97. 2012. 